\title{
PETROGRAFÍA Y GEOQUÍMICA DEL STOCK DE PAYANDÉ Y SU POSIBLE RELACIÓN CON EL MAGMATISMO JURÁSICO AL SUR DE COLOMBIA
}

\author{
July Quiceno-Colorado ${ }^{1}$; Susana Osorio-Ocampo²; Felipe Vallejo-Hincapié ${ }^{1,3}$; Andrés Salazar-Ríos ${ }^{1}$; \\ Cesar Augusto Ossa-Meza ${ }^{1}$; Leidy Giraldo-Alzate ${ }^{1}$; Larry Romero-Arboleda ${ }^{1}$
}

DOI: http://dx.doi.org/10.18273/revbol.v38n2-2016002 (c) (i) @

Forma de citar: Quiceno, J., Osorio-Ocampo, S., Vallejo, F., Salazar, A., Ossa, C.A., Giraldo, L., y Romero, L. 2016. Petrografía y geoquímica del Stock de Payandé y su posible relación con el magmatismo Jurásico al sur de Colombia. Boletín de Geología, 38(2): 39-53.

\section{RESUMEN}

Al oriente de la Cordillera Central Colombiana, en el municipio de San Luis-Tolima, afloran rocas ígneas plutónicas post-Triásico Tardío conocidas como Stock de Payandé. Los resultados de campo, petrográficos y geoquímicos revelan que estas rocas corresponden a un solo cuerpo con variaciones composicionales desde diorita gabróica a granodiorita, siendo ésta última la litología predominante en el área de estudio. Los diagramas de discriminación tectónica muestran que las rocas están asociadas a ambientes de arcos volcánicos formados en márgenes continentales activas. Las comparaciones químicas y posición de este intrusivo, permiten hacer una correlación a nivel regional con cuerpos plutónicos y volcano-sedimentarios reportados como Jurásicos en el Valle Superior del Magdalena (VSM) y en la Cordillera Central de Colombia.

Palabras clave: Stock de Payandé, Valle Superior del Magdalena, Cordillera Central, Arco Volcánico.

\section{PETROGRAPHY AND GEOCHEMISTRY OF PAYANDÉ STOCK AND ITS RELATION TO JURASIC MAGMATISM OF THE SOUTHERN COLOMBIA}

\begin{abstract}
Post-Triassic plutonic rocks, known as Payande Stock, are outcropping in the eastern flank of the Colombian Central Cordillera nearby San Luis-Tolima town. Detailed cartographic, petrographic, and geochemical analyses carried out on these rocks reveal compositional variations from gabbroic diorite to granodiorite. Tectonic discrimination diagrams show that the Payande Stock is associated with volcanic arcs formed in active continental margins. The chemical characteristics and fieldwork allow us to make a comparison between this intrusive with volcanic and plutonic regional bodies originated during the Jurassic in the Central Cordillera and Upper Magdalena Valley.
\end{abstract}

Keywords: Payande Stock, Upper Magdalena Valley, Jurassic, Central Cordillera, Volcanic Arcs.

\footnotetext{
${ }^{1}$ Universidad de Caldas, Departamento de Geología. Semillero de Investigación en Metalografía y Petrografía. quice19@hotmail.com.

2 Instituto de Geofísica, Unidad Michoacán, Universidad Nacional Autónoma de México.

${ }^{3}$ Instituto de Investigaciones en Estratigrafía (IIES)-Grupo de Investigación en Estratigrafía y Vulcanología -GIEV"CUMANDAY"-Universidad de Caldas.
} 


\section{INTRODUCCIÓN}

La zona de estudio está localizada en el flanco oriental de la Cordillera Central colombiana en límites con el Corregimiento de Payandé, Municipio de San Luis (Departamento del Tolima; FIGURA 1). En esta área afloran rocas ígneas plutónicas y subvolcánicas en contacto intrusivo con calizas fosilíferas de la Formación Payandé de edad Triásico Superior, haciendo parte del conjunto ígneo del basamento del Valle Superior del Magdalena (VSM) (Renz en Trumpy, 1943; Geyer, 1973). Aunque estas rocas no han sido ampliamente estudiadas, trabajos previos reportan granodioritas, cuarzodioritas y cuarzomonzonitas las cuales han sido denominadas como Stock de Payandé (Barrero, 1969; Núñez, 2001). Estos mismos estudios, de acuerdo a inferencias meramente de campo, proponen que esta unidad hace parte de un importante evento magmático que tuvo lugar a principios del Mesozoico en esta región de Colombia.

Para incrementar el conocimiento en esta unidad, se realizó una revisión cartográfica y un muestreo a escala 1:10.000 de las rocas ígneas plutónicas y los diques asociados. Posteriormente se llevó a cabo un análisis petrográfico de los cuerpos ígneos. A partir de esto, cinco fracciones de roca fueron seleccionadas para los análisis químicos de roca total, elementos mayores y traza. Con estos resultados, el trabajo tiene como objetivo caracterizar petrográfica y geoquímicamente las rocas ígneas que conforman el Stock de Payandé, a la altura del sector Río Frío (FIGURA 1). Esta nueva información permitirá realizar una comparación geoquímica con unidades geológicas de edades similares, evaluando los modelos tectono-magmáticos existentes para la zona.
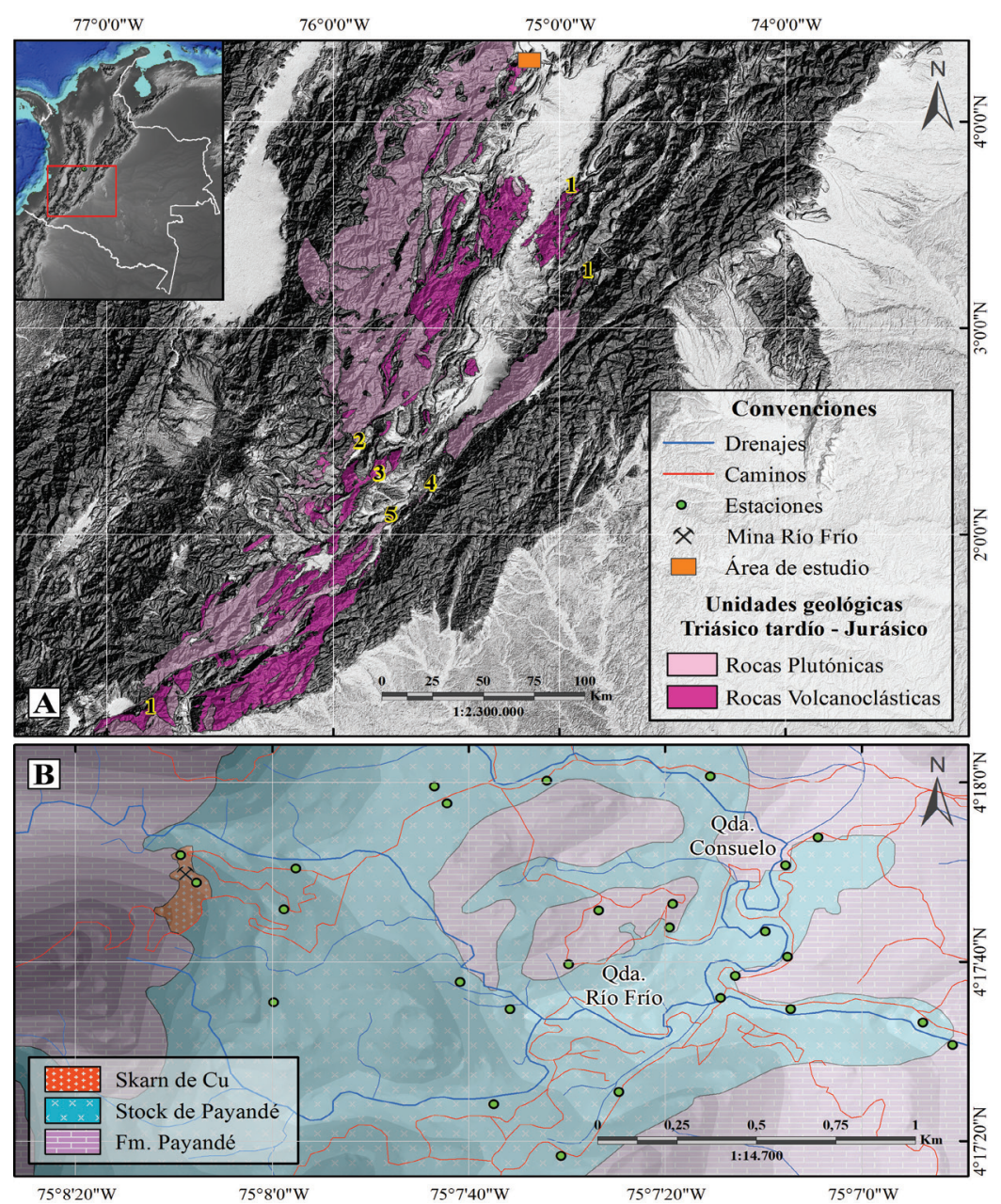

FIGURA 1. Mapa geológico-localización del área estudiada. A. Distribución de unidades plutónicas y volcanoclásticas del Triásico Tardío-Jurásico en el VSM (Gómez et al., 2007). B. Cartografía geológica detallada de la zona de estudio. Tomado y modificado de Barrero (1969). Los datos geoquímicos usados en la comparación provienen de (1) Fm. Saldaña (Narváez, 2011; Villagómez et al., 2011); (2) Batolito de Ibagué (Bustamante et al., 2010); (3) Monzodiorita de las Minas (Bustamante et al., 2010); (4) Granito de Garzón (Bustamante et al., 2010); (5) Monzogranito de Altamira (Bustamante et al., 2010). 


\section{CONTEXTO GEOLÓGICO}

Diversos trabajos han contribuido al conocimiento geológico de las cuencas interandinas colombianas a la altura del VSM durante el Mesozoico (Hubach, 1957; Cediel et al., 1980; Mojica y Macia, 1982; Núñez, 1986, 2001; Toussaint and Restrepo, 1994; Bayona et al., 1994, 2005; 2006; Mojica et al., 1996; Sarmiento-Rojas et al., 2006; Vásquez et al., 2006; Bustamante et al., 2010; Spikings et al., 2014). Todas las rocas aflorantes en este sector son agrupadas dentro del Terreno Chibcha según Toussaint and Restrepo (1994). El relleno sedimentario del VSM consta de rocas sedimentarias marinas, tobas, capas rojas y depósitos piroclásticos, las cuales reflejan periodos de vulcanismo subaéreo con algunas incursiones marinas durante el Mesozoico (Mojica y Macia, 1982; Bayona et al., 1994; Mojica et al., 1996) (FIGURA 1). Estas unidades se encuentran en contacto intrusivo y discordante con cuerpos batolíticos de composición intermedia-ácida con edades Triásico-Jurásico, las cuales están distribuidas a lo largo del VSM, Cordilleras Central y Oriental (Núñez, 1986; Jaillard et al., 1990; Toussaint, 1995; Ramos and Alemán, 2000; Núñez, 2001; Cediel et al., 2003; Bustamante et al., 2010; Spikings et al., 2014) (FIGURA 1).

Durante El Triásico Tardío y el Jurásico Temprano, un incremento en el magmatismo continental ha sido reportado por varios autores (Mojica y Macia, 1982; Núñez, 1986, 2001; Bayona et al., 1994; Mojica et al., 1996; Sarmiento-Rojas et al., 2006; Bustamante et al., 2010; Spikings et al., 2014). El ambiente tectónico de formación de este evento, sin embargo, es aún controversial. Algunos autores consideran que para el Triásico-Jurásico, la sedimentación volcanoclástica estuvo asociada a cuencas extensionales y el magmatismo fue generado en ambientes de rifting relacionados al rompimiento de Pangea (Pindell and Dewey, 1982; Mojica y Macia, 1982; Jaillard et al., 1990; Cediel et al., 2003; Pindell and Keenan, 2010). Por otra parte, trabajos como Bayona et al. (1994); McCourt et al. (1984); Núñez, (1986); Toussaint, (1995); Vásquez et al. (2006); Sarmiento-Rojas et al. (2006); Bustamante et al. (2010); Spikings et al. (2014) proponen un ambiente de arco magmático relacionado a una zona de subducción y cuencas sedimentarias tras-arco para las rocas Mesozoicas expuestas en el VSM. No obstante, estas interpretaciones son discutidas debido a que datos paleomagnéticos demuestran que algunos de estos cuerpos experimentaron movimientos hacia el norte en el Jurásico (Bayona et al., 2006).

Los avances en el conocimiento geológico del área de estudio son escasos y están restringidos a caracterizaciones de las mineralizaciones generadas durante la intrusión del Stock de Payandé en las calizas, dando origen al Skarn de la Mina Río Frío cerca de Payandé, Tolima (Alvarán et al., 2011). Con respecto a las unidades plutónicas aflorantes, no se conocen resultados publicados diferentes a Barrero (1969) y Núñez (2001). De acuerdo a estos trabajos, el Stock de Payandé está compuesto por un cuerpo de composición cuarzodiorítica intruido por otro de composición granodiorítica. Según Nelson (1959) y Barrero (1969) estas rocas fueron formadas entre finales del Jurásico y principios del Cretácico de acuerdo a la relación intrusiva del Stock de Payandé sobre la Formación Payandé de edad Triásico Superior (FIGURA 1).

\section{RESULTADOS}

\section{Relaciones de Campo}

Las rocas ígneas aflorantes en el área de interés exhiben poca deformación tectónica y en general moderada a baja alteración hidrotermal (FIGURA 2). Las litologías encontradas en la zona corresponden en su mayoría a rocas plutónicas ácidas e intermedias y en menor proporción a rocas básicas. Estas litologías están en contacto intrusivo con las calizas de la Formación Payandé. Aunque los efectos de metamorfismo térmico producidos por las rocas plutónicas no son muy comunes macroscópicamente, con excepción del sector de la Mina Río Frío, dónde se observa una amplia zona de reacción conocida como el Skarn de Payandé (Alvarán et al., 2011). Como un último evento, cortando las unidades más antiguas, se observan diques porfídicos ácidos y básicos de dimensiones milimétricas a centimétricas (FIGURA 2A). Trabajos previos (Barrero, 1969) han señalado un contacto intrusivo entre las rocas plutónicas ácidas e intermedias, sin embargo, en el área de estudio no se encontraron evidencias de intrusión entre estos cuerpos. Macroscópicamente las rocas intrusivas varían de color entre gris claro a gris oscuro con textura fanerítica de grano medio a grueso (FIGURA 2). Los minerales reconocidos en campo consisten en feldespato, cuarzo y anfíboles principalmente. 

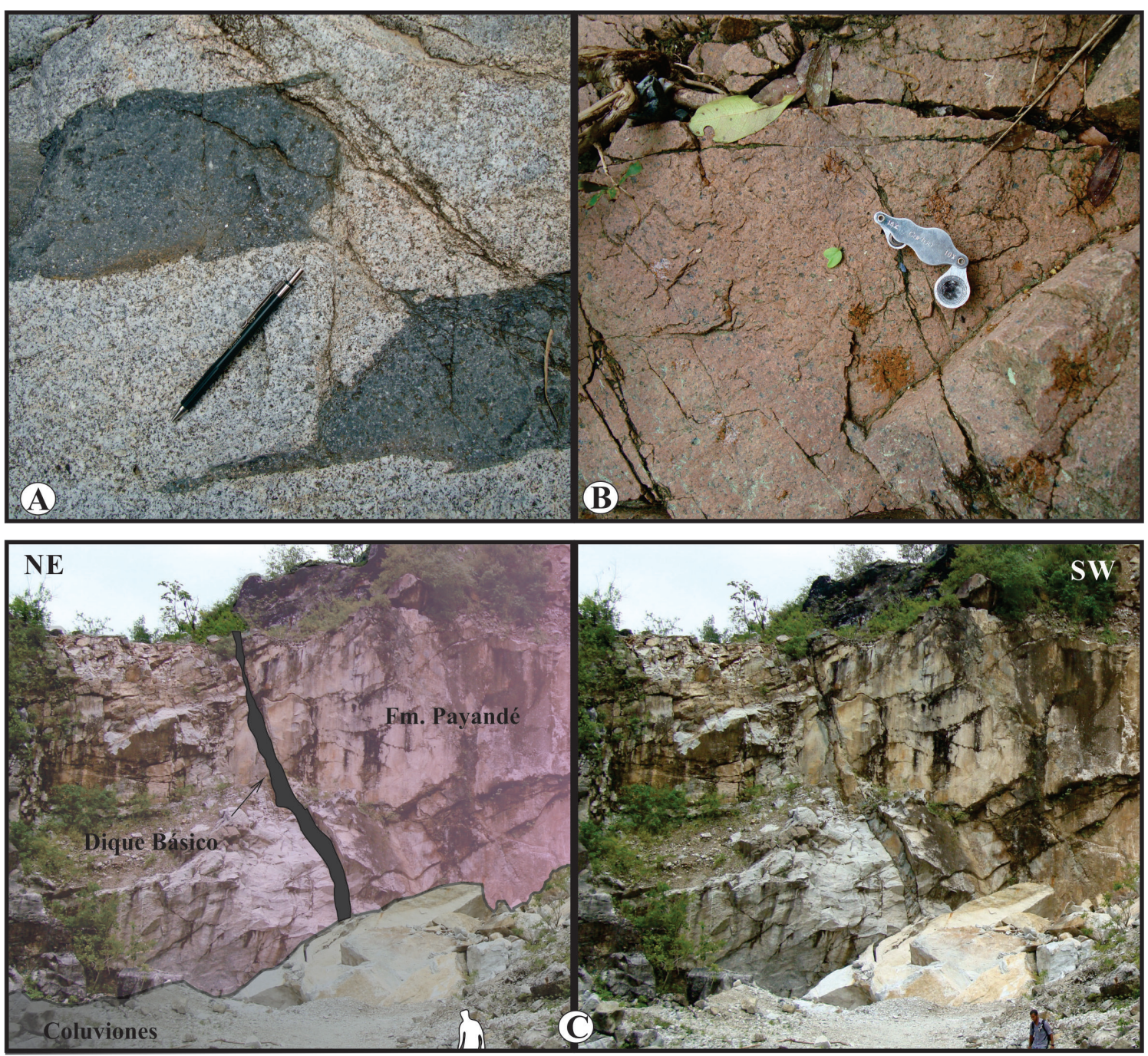

FIGURA 2. Aspectos del Stock de Payandé. A. Diques básicos intruyendo al Stock de Payandé B. Diques ácidos de coloración rojiza. C. Contacto intrusivo entre diques básicos y la Fm. Payandé.

\section{ANÁLISIS PETROGRÁFICO}

\section{Facies cuarzodirítica-tonalítica}

Ésta es la facies más abundante en el área de estudio (FIGURAS 1 y 2). El estudio de campo revela que el grado de meteorización es bajo, sin embargo, en los sectores cercanos al Skarn de Payandé la meteorización puede ser alta. Al microscopio, las rocas fueron clasificadas como cuarzodiorita-tonalita (Le Maitre, 2002) con textura general holocristalina fanerítica inequigranular, hipidiomórfica y texturas especiales poiquilíticas de apatito y circón en biotitas (FIGURA 3A-D). La plagioclasa es el mineral más abundante (43-57.4\%) con tamaños $>3 \mathrm{~mm}$ y presenta formas tabulares con zonación normal y macla tipo albita (FIGURA 3A, C). La composición de este feldespato es $\mathrm{An}_{39-47}$ y fue calculada a través de Michel-Levy.
Los minerales de alteración más comunes que se observaron en las secciones delgadas fueron sericita y saussurita (FIGURA 3D). Los cristales de cuarzo, con una abundancia entre $6 \%$ y $25 \%$, se presentan en formas irregulares rellenando intersticios con tamaños $>1 \mathrm{~mm}$ (FIGURA 3C-D). Como minerales accesorios se encuentran principalmente hornblenda y biotita (A-D). La hornblenda (4-20\%) exhibe formas euhedrales-anhedrales, coloraciones verde claro a café y tamaños $<3 \mathrm{~mm}$. Este mineral presenta en algunas ocasiones maclas simples y en algunos sectores se altera a clorita (FIGURA 3C). La mica biotita con abundancias hasta $17,2 \%$ presenta color marrón y formas subhedrales a anhedrales (FIGURA 3D). Apatito, circón, esfena y opacos igualmente se encuentran como minerales accesorios aunque en menor proporción. 


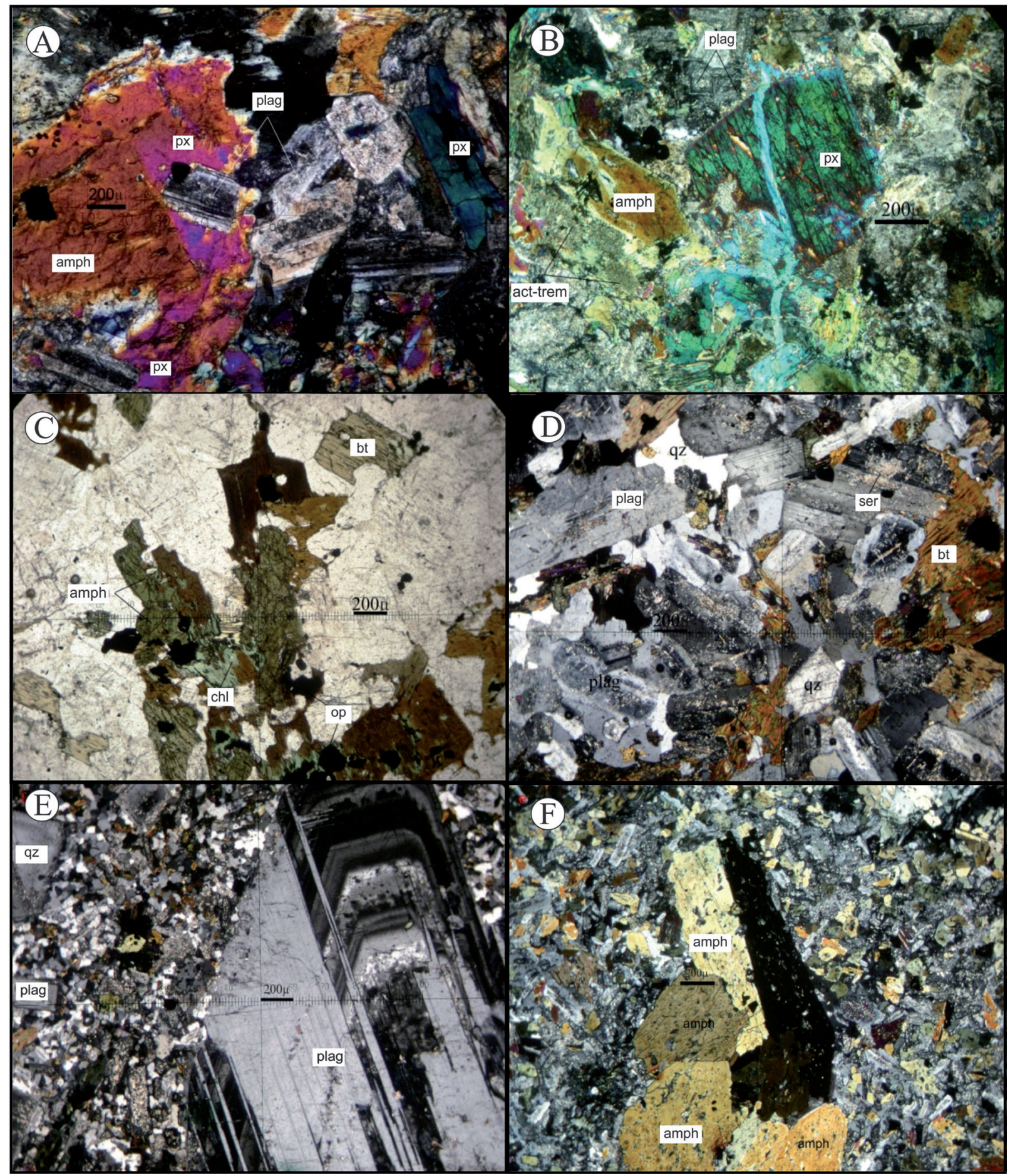

FIGURA 3. Microfotografía de las rocas plutónicas: A. Textura holocristalina fanerítica desarrollada por los piroxenos (px) y plagioclasas (plag) (Imagen con analizador). B. Piroxeno euhedral alterado a actinolita-tremolita (act-trem) (Imagen con analizador). C. Minerales accesorios biotita (bt), opacos (op) y hornblendas (amph) alterándose a clorita (chl) (Imagen sin analizador). D. Cuarzo-biotita-plagioclasa, asociación mineral predominante en la facies ácidas (Imagen con analizador). E. Fenocristales de cuarzo (qz) y plagioclasa (plag) con zonamiento (Imagen con analizador). F. Textura glomeroporfirítica de anfíboles (amph). 


\section{Facies gabróica}

Esta facies aparece en un pequeño sector al suroriente de la zona de estudio y en campo exhibe un grado de alteración moderado a alto. El análisis petrográfico revela una textura general holocristalina fanerítica, inequigranular, hipidiómorfica, con tamaños de los cristales de fino a medio, clasificada como Piroxenita Hornbléndica (Le Maitre, 2002). El mineral más abundante en esta facies es el clinopiroxeno con porcentajes del 22 al 30\% (FIGURA 3B). Formas subhedrales-anhedrales son características importantes de los minerales, así como la alteración actinolitatremolita presentes en los bordes de los cristales (FIGURA 3A-B). La plagioclasa (20-28\%) es otro mineral primario observado en esta facies, sus formas son subhedrales, con tamaños $<1 \mathrm{~mm}$ y presentan maclas tipo albita y zonación normal. Esta fase mineral está fuertemente alterada a saussurita y epidota. Dentro de los minerales accesorios, cristales de horblenda (13 -18\%) muestran coloraciones verde y café, hábitos prismáticos y tamaños $<2 \mathrm{~mm}$. Minerales como epidota y clorita son productos de alteración de los anfíboles (FIGURA 3A-B).

\section{Cuerpos subvolcánicos (diques)}

De acuerdo al estudio de campo, los diques corresponden a cuerpos subvolcánicos moderadamente alterados que registran el último pulso magmático en la zona (FIGURA 2C). Estos diques atraviesan las facies previamente descritas y en algunos casos se observa el contacto directo con las calizas de la formación Payandé (FIGURA 2). La composición de los cuerpos subvolcánicos varía entre microgabros, microdioritas y microcuarzodioritas (Le Maitre, 2002.). Al microscopio, estas rocas muestran una textura general porfídica con texturas especiales glomeroporfídica de anfíboles y poiquilítica de apatito en biotitas (FIGURA 3E-F). Los fenocristales identificados en su mayoría son plagioclasa con una composición An $<50$ calculada según el método Michelle Levy (FIGURA 3E). Los cristales de feldespato se encuentran alterados a sericita y en algunos casos reemplazados por calcita. La zonación normal de las plagioclasas es una característica muy común en sección delgada, aunque la zonación oscilatoria fue vista en algunos cristales (FIGURA 3E). Otros componentes importantes en los fenocristales son cristales de biotitas y anfíboles, los cuales están igualmente alterados a calcita, clorita y epidota principalmente. La presencia de cuarzo en la asociación de los fenocristales es notoria, alcanzando en ocasiones hasta un 16\% (FIGURA 3E). Por lo general esta fase mineral se identificó con formas irregulares y con tamaños que no superaban los $8 \mathrm{~mm}$ (FIGURA 3E). En cuanto a la masa fundamental los tamaños de los cristales varían entre 0,01 y $0,2 \mathrm{~mm}$ (FIGURA 3F). Entre los microcristales observados están la plagioclasa, anfíbol y cuarzo, los cuales forman texturas microlítica e intergranular (FIGURA 3E-F). En el sector de la quebrada Río Frío, se observaron diques de color rojizo los cuales habían sido reportados en estudios anteriores como diques máficos con pátinas de hierro (Figura 2B). Bajo el microscopio, sin embargo, estas rocas presentan textura general holocristalina porfirítica con fenocristales de cuarzo, plagioclasa, anfíbol y en muy baja proporción minerales opacos y feldespato potásico. Es de aclarar que ésta asociación presenta una muy alta alteración a saussurita y clorita. Respecto a la masa fundamental se identificaron principalmente minerales de plagioclasa y cuarzo como principales y anfíbol como accesorio. No obstante, el alto grado de meteorización en esta roca no permitió describir con mayor detalle la fracción más pequeña.

\section{Geoquímica de elementos mayores, traza $y$ tierras raras.}

Los análisis geoquímicos se realizaron sobre la suite de rocas que componen el cuerpo principal del Stock de Payandé a los alrededores de la Mina Río Frío. En total se analizaron cinco rocas, procurando que tuviesen la mayor representatividad de las diferentes composiciones del cuerpo ígneo. Los resultados muestran que el contenido de sílice de las rocas varía desde 52,28 hasta $65,8 \%$ en peso (TABLA 1). De acuerdo al diagrama TAS de Middlemost (1994) (FIGURA 4A) las muestras se clasifican como granodioritas (LAS05 y P14), diorita (PTO 16A y SM3) y diorita gabróica (E4). Todos los análisis se ubican en el campo de las rocas calcoalcalinas (FIGURA 4B), y de acuerdo al índice de Shand son metaluminosas (FIGURA 4C).

En los diagramas Harker se observa una correlación negativa de $\mathrm{MgO}, \mathrm{CaO}, \mathrm{FeO}$ y $\mathrm{TiO}_{2}$ con respecto a $\mathrm{SiO}_{2}$; el $\mathrm{Na}_{2} \mathrm{O}$ y el $\mathrm{K}_{2} \mathrm{O}$ aumentan progresivamente, mientras que el $\mathrm{Al}_{2} \mathrm{O}_{3}$ y el $\mathrm{P}_{2} \mathrm{O}_{5}$ presentan tendencias positivas, con una inflexión hacia una tendencia negativa aproximadamente a $57 \%$ de $\mathrm{SiO}_{2}$ (FIGURA 5). 
TABLA 1. Resultados de análisis químicos de elementos mayores en \% en peso y traza en ppm obtenidos de las muestras estudiadas. Las muestras fueron analizadas en los laboratorios Acme, Vancouver BC-Canadá a través de un espectrómetro de masas acoplado (ICP-MS). La precisión lograda por este procedimiento es de \pm 2 y \pm 5 para una concentración analítica entre 50 y 5 ppm respectivamente.

\begin{tabular}{|c|c|c|c|c|c|}
\hline Muestra & E4 & P16A & LAS05 & P14 & SM3 \\
\hline Símbolo & $\Delta$ & * & $\star$ & 0 & \& \\
\hline $\mathrm{SiO}_{2}$ & 52,28 & 56,54 & 65,89 & 64,5 & 57,84 \\
\hline $\mathrm{TiO}_{2}^{2}$ & 0,81 & 0,9 & 0,44 & 0,48 & 0,81 \\
\hline $\mathrm{Al}_{2} \mathrm{O}_{3}^{2}$ & 12,51 & 16,94 & 14,54 & 14,98 & 17,21 \\
\hline $\mathrm{Fe}_{2} \mathrm{O}_{3}^{3}$ & 8,85 & 5,73 & 3,96 & 3,4 & 7,36 \\
\hline $\mathrm{MnO}^{2}$ & 0,15 & 0,1 & 0,07 & 0,04 & 0,13 \\
\hline $\mathrm{MgO}$ & 10,14 & 4,3 & 1,95 & 1,83 & 3,42 \\
\hline $\mathrm{CaO}$ & 8,36 & 6,78 & 3,53 & 4,02 & 6,14 \\
\hline $\mathrm{Na}_{2} \mathrm{O}$ & 2,87 & 3,84 & 3,8 & 3,43 & 3,4 \\
\hline $\mathrm{K}_{2} \mathrm{O}$ & 1,59 & 1,62 & 2,43 & 3,31 & 1,77 \\
\hline $\mathrm{P}_{2}^{2} \mathrm{O}_{5}$ & 0,1 & 0,21 & 0,1 & 0,12 & 0,21 \\
\hline $\mathrm{S}^{25}$ & $<0,02$ & 0,76 & $<0,02$ & 0,05 & $<0,02$ \\
\hline LOI & 1,8 & 2,8 & 3,1 & 3,7 & 1,5 \\
\hline $\mathrm{Ba}$ & 670 & 491 & 569 & 677 & 655 \\
\hline $\mathrm{Rb}$ & 31,8 & 34,4 & 59,7 & 76,8 & 50,5 \\
\hline $\mathrm{Sr}$ & 873,1 & 582,8 & 407,6 & 362,1 & 511,4 \\
\hline $\mathrm{Zr}$ & 81,1 & 187,9 & 131,1 & 128,3 & 133,6 \\
\hline $\mathrm{Nb}$ & 3,6 & 7,6 & 6,4 & 6,3 & 7,1 \\
\hline $\mathrm{Ni}$ & 41,1 & 17,2 & 18,1 & 24 & 11,2 \\
\hline Co & 35 & 20,7 & 8,8 & 8,2 & 18,2 \\
\hline $\mathrm{Zn}$ & 26 & 35 & 102 & 107 & 107 \\
\hline $\mathrm{Cr}$ & 533 & 68 & 55 & 89 & 55 \\
\hline $\mathrm{La}$ & 7,2 & 17,8 & 12,3 & 18 & 18,6 \\
\hline $\mathrm{Ce}$ & 18,3 & 41 & 25,1 & 37,6 & 40,4 \\
\hline $\operatorname{Pr}$ & 2,41 & 4,99 & 2,86 & 4,03 & 4,83 \\
\hline $\mathrm{Nd}$ & 11 & 19,1 & 11 & 14,7 & 20,5 \\
\hline $\mathrm{Sm}$ & 2,6 & 3,93 & 2,21 & 2,54 & 3,95 \\
\hline $\mathrm{Eu}$ & 0,84 & 1,32 & 0,73 & 0,77 & 1,3 \\
\hline Gd & 3,17 & 3,81 & 2,3 & 2,43 & 3,9 \\
\hline $\mathrm{Tb}$ & 0,55 & 0,62 & 0,37 & 0,39 & 0,64 \\
\hline Dy & 3,19 & 3,41 & 2,14 & 2,17 & 3,4 \\
\hline Ho & 0,7 & 0,73 & 0,43 & 0,45 & 0,73 \\
\hline Er & 1,95 & 2,07 & 1,27 & 1,31 & 2,06 \\
\hline $\mathrm{Tm}$ & 0,32 & 0,33 & 0,2 & 0,19 & 0,32 \\
\hline $\mathrm{Yb}$ & 2,11 & 2,2 & 1,36 & 1,45 & 2,03 \\
\hline $\mathrm{Lu}$ & 0,3 & 0,34 & 0,22 & 0,21 & 0,32 \\
\hline Y & 19,7 & 20,3 & 13,1 & 12,7 & 20,4 \\
\hline Cs & 1 & 1,5 & 1,7 & 1,7 & 1,6 \\
\hline Тa & 0,2 & 0,5 & 0,7 & 0,6 & 0,5 \\
\hline Hf & 2,5 & 4,7 & 3,3 & 3,9 & 3,7 \\
\hline $\mathrm{Ga}$ & 14 & 18 & 14,6 & 13,7 & 17,9 \\
\hline $\mathrm{Sn}$ & 1 & 2 & 2 & 1 & 1 \\
\hline Th & 2,2 & 6 & 10,4 & 14,2 & 5,6 \\
\hline $\mathrm{U}$ & 0,5 & 1,6 & 2,5 & 3,5 & 1,4 \\
\hline V & 242 & 169 & 73 & 81 & 137 \\
\hline W & 0,25 & 0,8 & 1 & 0,7 & 1 \\
\hline Mo & 0,1 & 1,8 & 0,9 & 0,5 & 1,2 \\
\hline $\mathrm{Cu}$ & 52,5 & 93,9 & 7,8 & 11,4 & 35,1 \\
\hline $\mathrm{Pb}$ & 1,9 & 3,1 & 5,6 & 3,2 & 2,8 \\
\hline As & 3,9 & 2,8 & 0,6 & 1 & 1,1 \\
\hline $\mathrm{K} / \mathrm{Rb}$ & 424,57 & 402,78 & 353,59 & 372,26 & 296,02 \\
\hline $\mathrm{Rb} / \mathrm{Zr}$ & 0,39 & 0,18 & 0,46 & 0,6 & 0,38 \\
\hline $\mathrm{Nb} / \mathrm{Yb}$ & 1,71 & 3,45 & 4,71 & 4,35 & 3,5 \\
\hline $\mathrm{Nb} / \mathrm{Th}$ & 1,64 & 1,27 & 0,61 & 0,44 & 1,27 \\
\hline$(\mathrm{La} / \mathrm{Yb}) \mathrm{N}$ & 2,45 & 5,80 & 6,49 & 8,90 & 6,57 \\
\hline$(\mathrm{La} / \mathrm{Sm}) \mathrm{N}$ & 1,79 & 2,92 & 3,59 & 4,57 & 3,04 \\
\hline$(\mathrm{Ga} / \mathrm{Yb}) \mathrm{N}$ & 1,24 & 1,43 & 1,40 & 1,39 & 1,59 \\
\hline (Ti/Yb)N-MORB & 5,54 & 5,95 & 4,72 & 4,86 & 5,73 \\
\hline $\mathrm{Eu} / \mathrm{Eu}^{*}$ & 0,89 & 1,03 & 0,98 & 0,93 & 1,00 \\
\hline
\end{tabular}

En los elementos traza, las rocas del Stock de Payandé muestran bajos valores de elementos compatibles, tales como $\mathrm{Cr}$ (55-89 ppm) y Ni (11,2 - 41,1 ppm), a excepción de los valores de $\mathrm{Cr}$ de la muestra E4 (533 ppm); los valores de V son consistentemente más bajos conforme aumenta el $\mathrm{SiO}_{2}$ (TABLA 1).

En los diagramas multielementos, las tierras raras livianas (LREE) se encuentran enriquecidas con respecto a las tierras raras pesadas (HREE), obteniéndose una relación $\mathrm{La} / \mathrm{Yb}_{\mathrm{N}}$ entre 2,45 (diorita gabróica) hasta 8,9 (granodiorita). Las LREE están enriquecidas 2580 veces los valores de condrita (FIGURA 6A). Las HREE muestran en general una ligera fraccionación $\left(\mathrm{Gd} / \mathrm{Yb}_{\mathrm{N}}=1,24-1,59\right)$. No se presentan anomalías de $\mathrm{Eu}$ considerables $\left(\mathrm{Eu} / \mathrm{Eu}^{*} \sim 1\right)$ únicamente se observa una pequeña anomalía negativa en la muestra de gabronorita (ver TABLA 1). En el diagrama normalizado a manto primitivo (FIGURA 6B) se evidencia un enriquecimiento en LILE (e.g. Cs, Rb, Th, K) con respecto a los elementos HFSE (e.g. Nb, Ta, Ti, P), presentando consistentes anomalías positivas de $\mathrm{Th}-\mathrm{U}, \mathrm{K}, \mathrm{Pb}$ y $\mathrm{Sr}$, así como una leve anomalía positiva de $\mathrm{Zr}$ en algunas muestras. Asimismo, se observan constantes anomalías negativas de Nb-Ta, La-Ce, Pr, P y Ti.

Las tendencias observadas en los diagramas de normalización, tanto de tierras raras como de elementos traza, así como las anomalías negativas de $\mathrm{Nb}$ y Ta sugieren una firma geoquímica de márgenes convergentes (e.g. McCulloch and Gamble, 1991; Hawkesworth et al., 1994; Walker et al., 2001; Long et al., 2001). Las características geoquímicas de las rocas en este estudio guardan similitud en los elementos mayores, LILE y REE con los cuerpos producidos por subducción en los Andes Centrales (e.g. Batolito Costero de Perú, Pitcher et al., 1985). Las altas relaciones Ta/ $\mathrm{Yb}(0,09-0,5), \mathrm{Th} / \mathrm{Yb}(1,09-9,79)$, así como las bajas relaciones $\mathrm{Sc} / \mathrm{Ni}(0,42-1,52)$ son congruentes con rocas de márgenes continentales activas (Bailey, 1981; Pearce, 1983). Esta interpretación está reforzada por los diagramas de discriminación tectónica, que indican igualmente ambientes de arco magmático (FIGURA 7).

El comportamiento relativamente plano de las tierras raras pesadas HREE $\left(\mathrm{Gd} / \mathrm{Yb}_{\mathrm{n}}=1,24-1,59\right.$; FIGURA 6; TABLA 1), sugiere que el magma formador las rocas proviene de un fundido somero localizado a una profundidad menor de $80 \mathrm{~km}$ con ausencia de granate residual (Johnson, 1994; Hirschmann and Stolper, 1996; Van Westrenen et al., 2001; Booden et al., 2011; Ordóñez et al., 2011). La ausencia de anomalías de Eu indica la cristalización tardía de la plagioclasa debido a que no 
fue fraccionada durante el ascenso a la superficie (Siebe et al., 2004) o por una condición alta de fugacidad del oxígeno $\left(f \mathrm{O}_{2}\right)$ donde el Eu se mantiene en estado $\mathrm{Eu}^{+3} \mathrm{y}$ no entra en la estructura de la plagioclasa (Hezarkhani, 2006; Wilson, 2007).

\section{(A)}

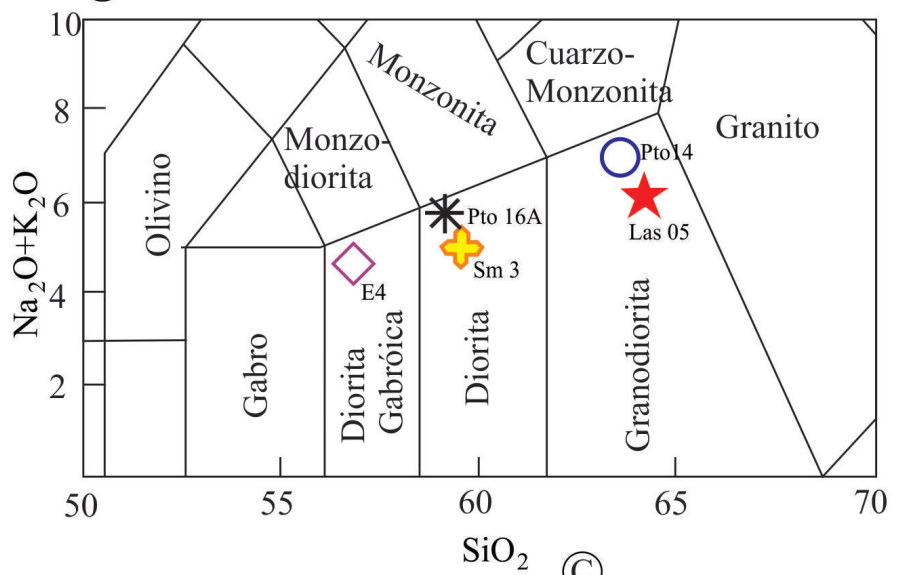

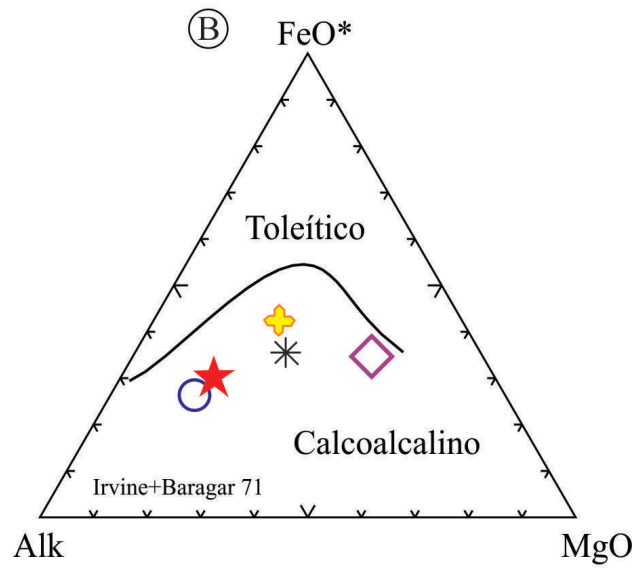

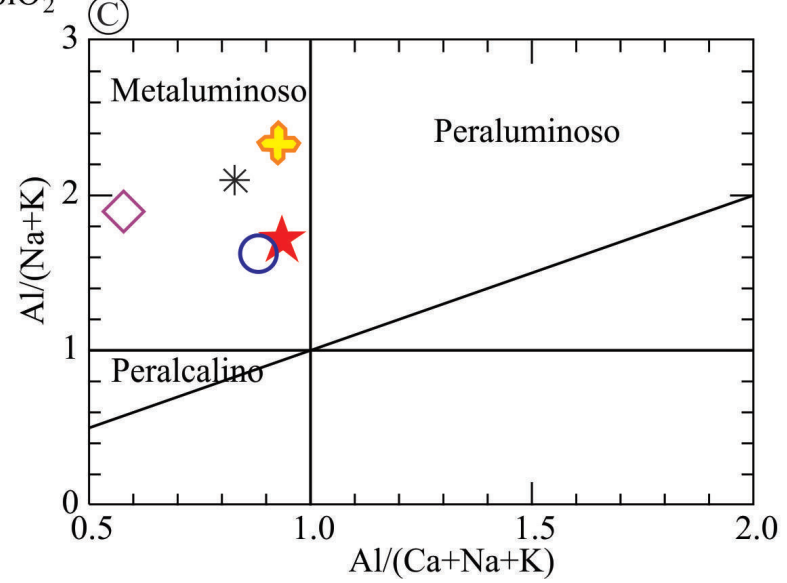

FIGURA 4. A. Clasificación química de las rocas con base en el diagrama TAS para rocas plutónicas (Middlemost, 1994). B. Diagrama AFM propuesto por Irvine and Baragar (1971). C. Índice de Shand de Maniar and Piccoli (1989).
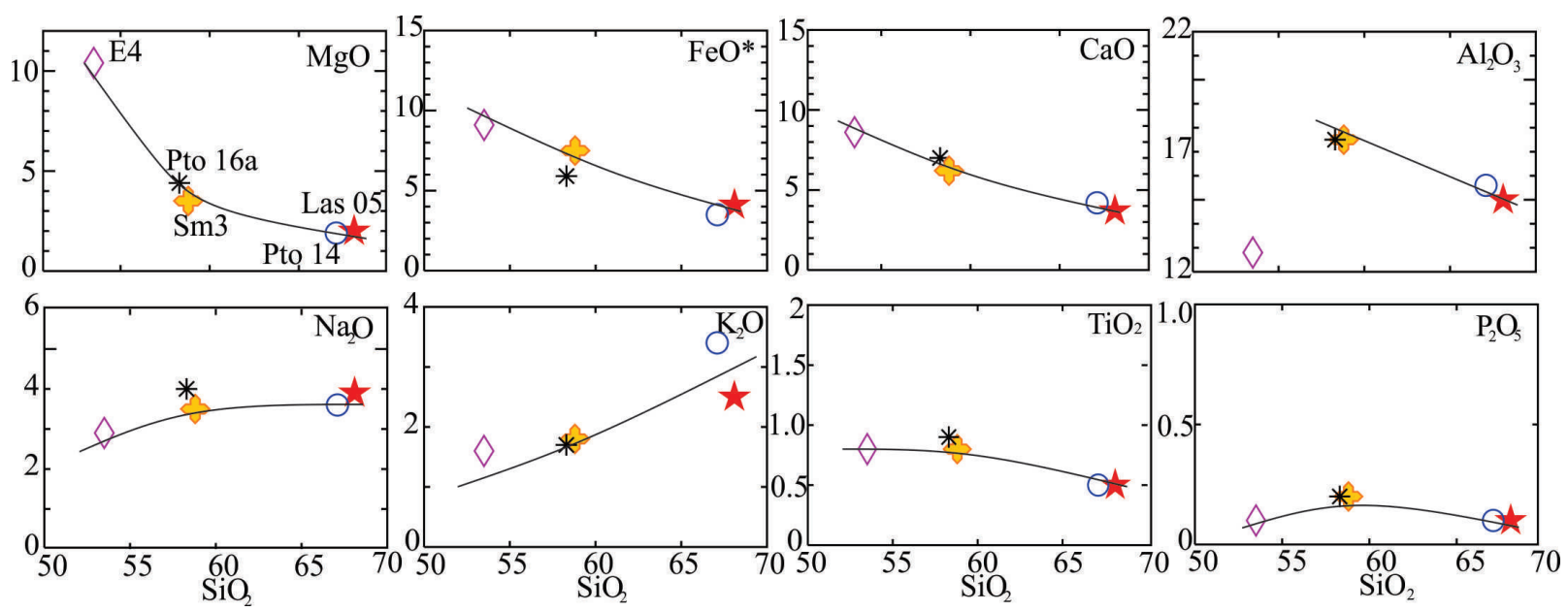

FIGURA 5. Diagramas de variación de los elementos mayores en porcentaje en peso contra el contenido de $\mathrm{SiO}_{2}$. Los patrones indican cristalización fraccionada del magma. 

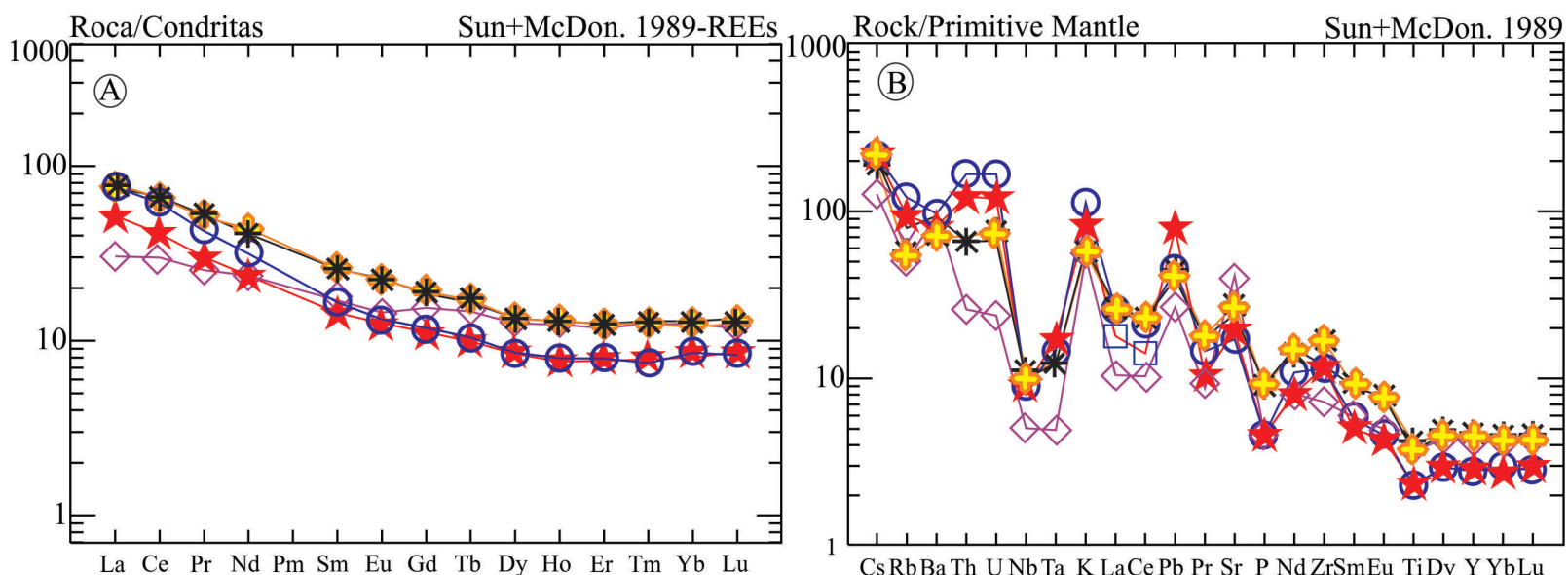

FIGURA 6. A. Condrita normalizada con la composición de las tierras raras en ppm (Sun and McDonough, 1989) de las rocas analizadas en la zona de estudio. B. Distribución de los elementos traza normalizado con los valores de manto primitivo de Sun and McDonough (1989)

(A)

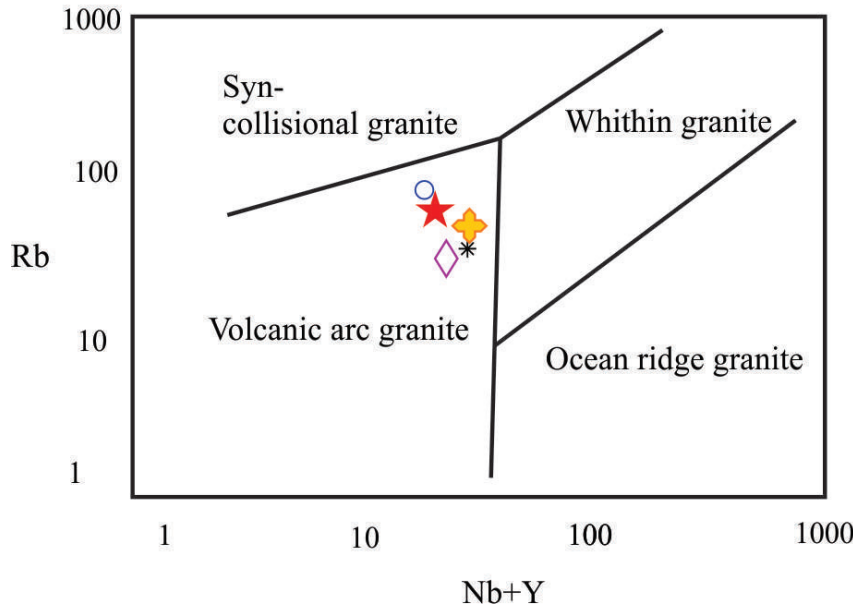

(B)

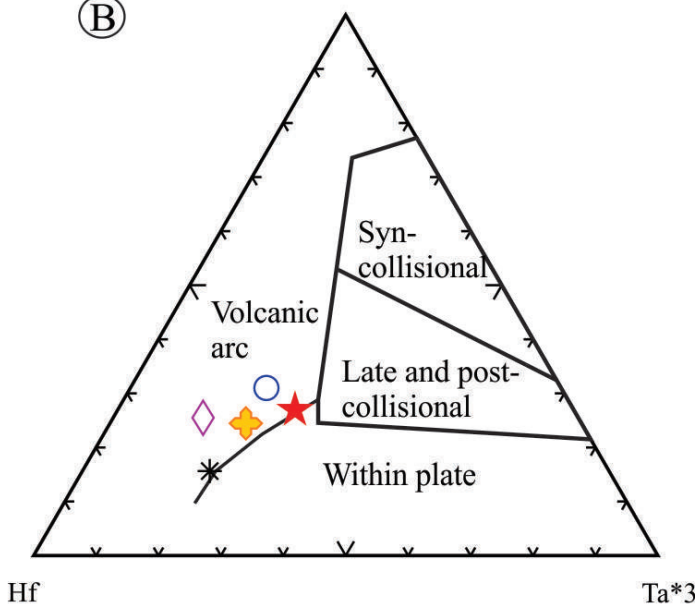

FIGURA 7. Diagramas de discriminación tectónica A. Diagrama propuesto por Pearce et al. (1984). B. Diagrama de discriminación Hf - Rb/30 - Ta*3 para granitos (Harris et al., 1986).

A pesar de que el comportamiento de los elementos mayores en los diagramas Harker sugieren una cristalización fraccionada, otras características geoquímicas indican que este proceso no es el único causante de la variedad composicional de las rocas del Stock de Payandé. Las relaciones $\mathrm{K} / \mathrm{Rb}$ y $\mathrm{Rb} / \mathrm{Zr}$ no cambian significativamente con los procesos de cristalización fraccionada, por esto, las variaciones en estas relaciones deben ser interpretados como indicativos de contaminación cortical (Davidson et al., 1988). Para este estudio, dichas relaciones varían moderadamente $(\mathrm{K} / \mathrm{Rb}=296,02-424,57 ; \mathrm{Rb} / \mathrm{Zr}=$ 0,18 - 0,6). Para comprobar dichas tendencias, se realizaron gráficos de discriminación entre procesos de cristalización fraccionada y contaminación/asimilación (FIGURA 8). De acuerdo al gráfico de elementos incompatibles propuesto por Davidson et al. (1988), la muestra de diorita gabróica (E4) presenta una tendencia afín con un sistema cerrado de cristalización fraccionada (FIGURA 8A); sin embargo este no es el caso de las cuarzodioritas y granodioritas, pues éstas presentan una tendencia intermedia entre cristalización fraccionada y cristalización fraccionada/asimilación (AFC). Este patrón, contrario a un sistema cerrado de cristalización fraccionada también es detectado en diagramas con elementos mayores (FIGURA 8B), o en diagramas que usan trazas inmóviles, como el $\mathrm{Nb} / \mathrm{Yb}$ vs $\mathrm{Th} / \mathrm{Yb}$ (FIGURA 8C). 


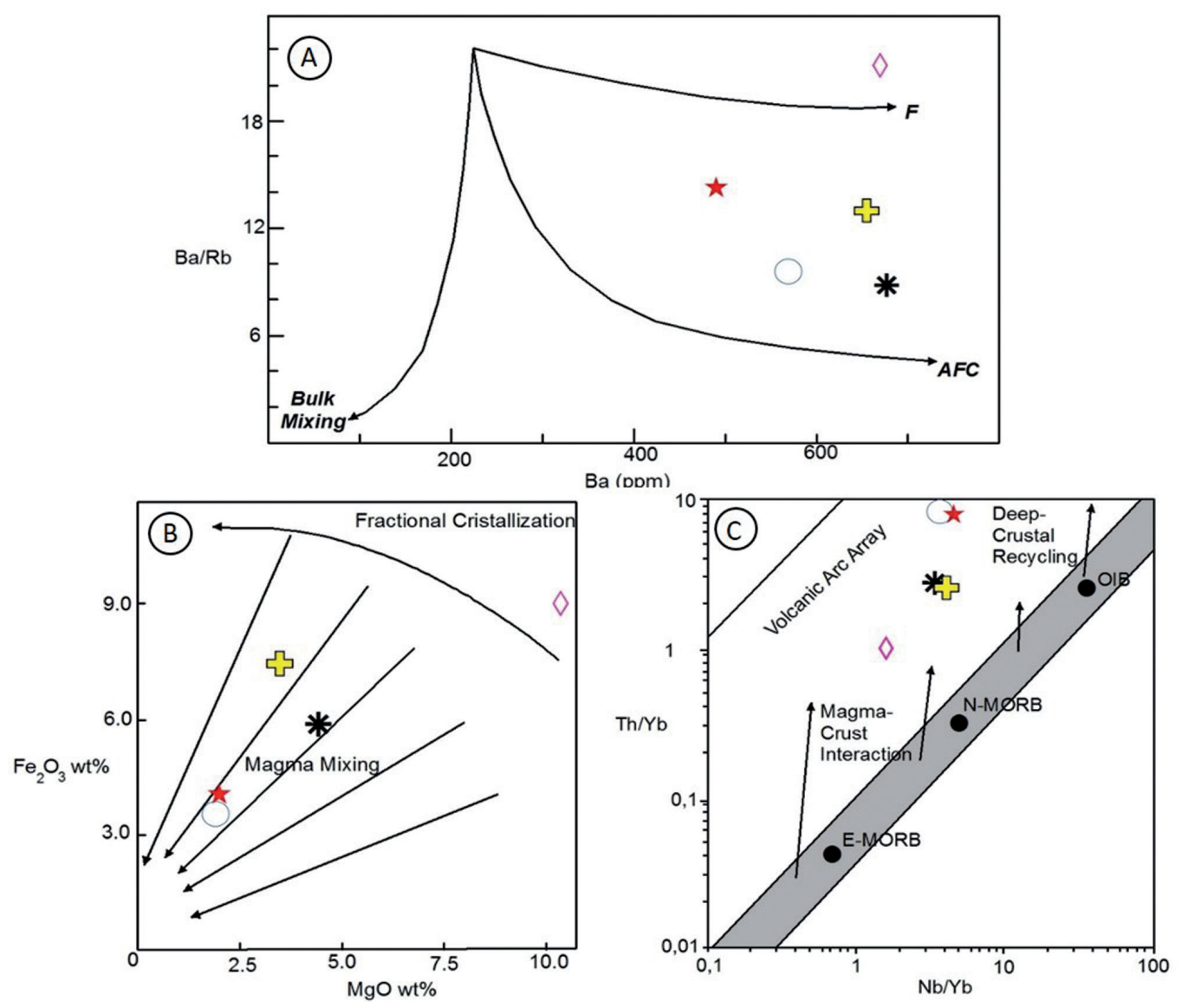

FIGURA 8. A. Diagrama Ba vs Ba/Rb propuesto por Davidson et al. (1988). F: Cristalización Fraccionada. AFC: Asimilación y Cristalización Fraccionada. B. Diagrama propuesto por Yang et al. (2015) para identificar la contaminación de los magmas. C. Diagrama $\mathrm{Nb} / \mathrm{Yb}$ vs $\mathrm{Th} / \mathrm{Yb}$ propuesto por Pearce (2008). En general, la gabronorita está asociadas a cristalización fraccionadas, mientras las rocas intermedias y ácidas poseen un componente de contaminación. Símbolos como en la TABLA 1.

\section{DISCUSIÓN Y CONCLUSIÓN}

Las evidencias de campo, petrográficas y geoquímicas revelan que el Stock de Payandé consta de un solo cuerpo plutónico con variaciones litológicas desde rocas básicas a rocas ácidas, las cuales están intruyendo a rocas calcáreas de la Formación Payandé de edad Triásico. Si bien diversos autores han señalado que las coloraciones rojizas en estas rocas se deben a pátinas ferruginosas, al microscopio no se observaron tales minerales que pudieran causar este fenómeno (FIGURAS 2 y 3). Por otro lado, los resultados geoquímicos demuestran que este cuerpo se formó en un arco magmático sujeto a cambios composicionales debido a procesos de diferenciación magmática, asimilación cortical y posiblemente mezcla de magmas.

Aunque no se cuenta con una edad radiométrica para el Stock de Payandé, las relaciones de campo sugieren que este cuerpo podría hacer parte de uno de los eventos intrusivos del Batolito de Ibagué (177,3+/-8 Ma), el cual es considerado como un cuerpo plutónico de un arco magmático existente durante el Jurásico (Altenberger and Concha, 2005; Spikings et al., 2014). Esto permite inferir que el Stock de Payandé representa una de las unidades más septentrionales dentro de la franja de cuerpos Jurásicos con una firma geoquímica de arco descritos hasta el momento (FIGURA 1).

Con el fin de sustentar esta propuesta se realizó una comparación geoquímica con diferentes unidades plutónicas y volcano-sedimentarias asociadas genética y temporalmente al Stock de Payandé (e.g. Granito de Altamira, Diorita de las Minas, Batolito de Ibagué y Formación Saldaña - Bustamante et al., 2010; Narváez, 2011; Villagómez et al., 2011; Spikings et al., 2014; TABLA 2). 
July Quiceno-Colorado, Susana Osorio-Ocampo, Felipe Vallejo-Hincapié, Andrés Salazar-Ríos, Cesar Augusto Ossa-Meza, Leidy Giraldo-Alzate, Larry Romero-Arboleda

TABLA 2. Datos químicos usados para la correlación entre unidades geológicas de la franja Triásica-Jurásica en Colombia. Los elementos mayores se presentan en $\%$ en peso y traza en ppm.

\begin{tabular}{|c|c|c|c|c|c|c|c|c|}
\hline \multirow[t]{2}{*}{ Muestra } & \multirow{2}{*}{\multicolumn{3}{|c|}{$\begin{array}{c}\text { Narváez (2011) } \\
\text { Fm. Saldaña }\end{array}$}} & \multirow{3}{*}{$\begin{array}{c}\begin{array}{c}\text { Villagómez } \\
\text { et al. (2011) }\end{array} \\
\text { Fm. Saldaña } \\
\bigcirc\end{array}$} & \multicolumn{4}{|c|}{ Bustamante et al. (2010) } \\
\hline & & & & & $\begin{array}{c}\text { Monzogranito } \\
\text { Altamira }\end{array}$ & $\begin{array}{l}\text { Diorita Las } \\
\text { Minas }\end{array}$ & $\begin{array}{l}\text { Batolito de } \\
\text { Ibagué }\end{array}$ & $\begin{array}{c}\text { Granito de } \\
\text { Garzón }\end{array}$ \\
\hline Símbolo & $\square$ & $\square$ & $\square$ & & $\square$ & њ & $\triangle$ & $\nabla$ \\
\hline $\mathrm{SiO}_{2}$ & 58,3 & 61,1 & 55,7 & 63,7 & 63,5 & 58,1 & 56,6 & 57,1 \\
\hline $\mathrm{TiO}_{2}^{2}$ & 0,6 & 0,4 & 1 & 0,5 & 0,6 & 0,9 & 0,9 & 0,8 \\
\hline $\mathrm{Al}_{2} \mathrm{O}_{3}$ & 17,1 & 17,8 & 16,9 & 16 & 15,6 & 17,2 & 17,8 & 18,7 \\
\hline $\mathrm{FeO}$ & 5,4 & 4,6 & 6,5 & 3,7 & 5 & 7,4 & 7,6 & 6,5 \\
\hline $\mathrm{MnO}$ & 0,1 & 0,2 & 0,1 & 0,1 & 0,1 & 0,1 & 0,1 & 0,1 \\
\hline $\mathrm{MgO}$ & 3,2 & 2,8 & 3,8 & 1,2 & 1,9 & 3 & 3,5 & 2,5 \\
\hline $\mathrm{CaO}$ & 3,3 & 2 & 4,5 & 2,9 & 3,8 & 6 & 6,5 & 4,7 \\
\hline $\mathrm{Na}_{2} \mathrm{O}$ & 6,2 & 8,1 & 3,6 & 4,3 & 3,7 & 3,7 & 4,1 & 4,9 \\
\hline $\mathrm{K}_{2}^{2} \mathrm{O}$ & 1,4 & 0,8 & 5,3 & 4 & 3,9 & 2,2 & 1,7 & 1,9 \\
\hline $\mathrm{P}_{2} \mathrm{O}_{5}$ & 0,3 & 0,3 & 0,5 & 0,1 & 0,2 & 0,3 & 0,3 & 0,4 \\
\hline LOI & 4,5 & 3,1 & 3,7 & & 1,3 & 0,8 & 0,7 & 2 \\
\hline Total & 100,2 & 101,1 & 101,4 & & 99,6 & 99,7 & 99,8 & 99,6 \\
\hline $\mathrm{Be}$ & 1 & 1 & 2 & & & & & \\
\hline $\mathrm{Sc}$ & 9 & 5 & 13 & 8,7 & 8 & 17 & 18 & 8 \\
\hline $\mathrm{V}$ & 87 & 51 & 114 & 76 & 90 & 150 & 147 & 106 \\
\hline $\mathrm{Cr}$ & & & & 9 & & & & \\
\hline Co & 12,9 & 8,7 & 12,8 & 7 & & & & \\
\hline $\mathrm{Ni}$ & 21 & 20 & 20 & 5 & & & & \\
\hline $\mathrm{Cu}$ & & & & 18 & & & & \\
\hline $\mathrm{Zn}$ & & & & 56 & & & & \\
\hline $\mathrm{Ga}$ & 18,1 & 18,9 & 18,4 & 15,7 & 17,9 & 17,7 & 18,7 & 22,5 \\
\hline $\mathrm{Rb}$ & 33,4 & 18,7 & 155,7 & 97,1 & 105,6 & 48,6 & 56,2 & 60,7 \\
\hline $\mathrm{Sr}$ & 403,5 & 438,4 & 675,2 & 526 & 664,8 & 609,8 & 637,2 & 1043 \\
\hline $\mathrm{Y}$ & & & & 18,6 & 21,7 & 21,3 & 17,2 & 13,2 \\
\hline $\mathrm{Zr}$ & 144,5 & 148 & 279,2 & 176 & 216,1 & 120,6 & 126 & 166,9 \\
\hline $\mathrm{Nb}$ & 5,8 & 5,2 & 11,6 & 5,6 & 18 & 6 & 5,6 & 8,8 \\
\hline $\mathrm{Sn}$ & 1 & 1 & 1 & & & & & \\
\hline Cs & 1,8 & 0,5 & 1,5 & 2,2 & 0,8 & 0,7 & 3 & 1,2 \\
\hline $\mathrm{Ba}$ & 308 & 248 & 1298 & 1436 & 943 & 780 & 675 & 770 \\
\hline $\mathrm{Hf}$ & 3,7 & 3,6 & 7,9 & 4,6 & 5,9 & 3,5 & 3,4 & 4,3 \\
\hline $\mathrm{Ta}$ & 0,3 & 0,2 & 0,6 & 0,3 & 1,1 & 0,4 & 0,3 & 0,4 \\
\hline W & 0,5 & 0,5 & 1,1 & & & & & \\
\hline $\mathrm{Pb}$ & & & & 10,4 & 3,9 & 2,5 & 1,3 & 2,8 \\
\hline $\mathrm{La}$ & 20,3 & 24,6 & 47,4 & 25,4 & 54,3 & 21,8 & 19,7 & 24,6 \\
\hline $\mathrm{Ce}$ & 45 & 50,8 & 103,9 & 47,9 & 98,1 & 45,7 & 41,1 & 51,1 \\
\hline $\operatorname{Pr}$ & 5 & 5,4 & 10,8 & 5,1 & 10,5 & 5,7 & 5,1 & 6,1 \\
\hline $\mathrm{Nd}$ & 17,3 & 19 & 41,2 & 18,2 & 38 & 23,2 & 22,4 & 25,8 \\
\hline $\mathrm{Sm}$ & 3,6 & 3,5 & 7,4 & 3,4 & 5,9 & 4,6 & 4,2 & 4,5 \\
\hline $\mathrm{Eu}$ & 1,1 & 1,1 & 1,6 & 0,9 & 1,4 & 1,3 & 1,2 & 1,4 \\
\hline Gd & 3,1 & 2,7 & 5,8 & 3 & 4,8 & 4,3 & 3,8 & 3,6 \\
\hline $\mathrm{Tb}$ & 0,5 & 0,4 & 0,9 & 0,5 & 0,7 & 0,7 & 0,6 & 0,5 \\
\hline Dy & 2,5 & 2,1 & 5 & 2,8 & 3,7 & 3,7 & 3,1 & 2,6 \\
\hline Ho & 0,5 & 0,4 & 1 & 0,6 & 0,8 & 0,8 & 0,6 & 0,5 \\
\hline Er & 1,4 & 1,2 & 2,7 & 1,9 & 2,2 & 2,1 & 1,8 & 1,2 \\
\hline $\mathrm{Tm}$ & 0,2 & 0,2 & 0,4 & 0,3 & 0,4 & 0,3 & 0,3 & 0,2 \\
\hline $\mathrm{Yb}$ & 1,3 & 1,2 & 2,7 & 1,9 & 2,2 & 2,1 & 1,8 & 1,2 \\
\hline $\mathrm{Lu}$ & 0,2 & 0,2 & 0,4 & 0,3 & 0,4 & 0,3 & 0,3 & 0,2 \\
\hline Th & 2,5 & 2,7 & 13,1 & 6,6 & 14,5 & 2,7 & 13,1 & 4,1 \\
\hline $\mathrm{U}$ & 0,8 & 0,7 & 3,5 & 1,9 & 4,1 & 0,7 & 2,1 & 1,9 \\
\hline
\end{tabular}


El gráfico de normalización a condrita muestra un enriquecimiento de LREE sobre HREE en las unidades correlacionables con el Stock de Payandé. Sin embargo, hay una tendencia general de estas unidades a ser más ricas en LREE $\left(\mathrm{La} / \mathrm{Yb}_{\mathrm{n}}=7,45-17,70\right)$, indicando que estás unidades se formaron en cámaras magmáticas con mayor diferenciación, o que la contaminación y asimilación cortical en estas unidades al sur es mayor (FIGURA 9A). El enriquecimiento de LILE sobre HFSE y las anomalías de $\mathrm{Nb}$ y Ta en estas unidades (FIGURA 9B), así como los diagramas de discriminación tectónica (FIGURA 9C-D) indican que la región de la Cordillera Central de Colombia se encontraba un ambiente de subducción. No se encontró ninguna evidencia geoquímica que sugiera ambientes distensivos o de rift para estas unidades, como ha sido propuesto para las cercanías del Stock de Payandé debido a la presencia de rocas que se interpretaron como alcalinas (Jaillard et al., 1990; Cediel et al., 2003; Pindell and Kennan, 2010).

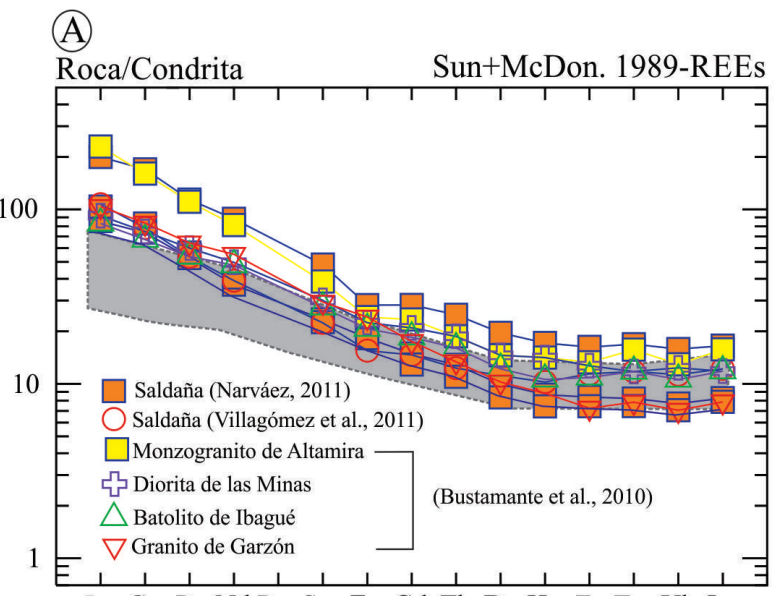

La Ce Pr Nd Pm Sm Eu Gd Tb Dy Ho Er Tm Yb Lu

(B)
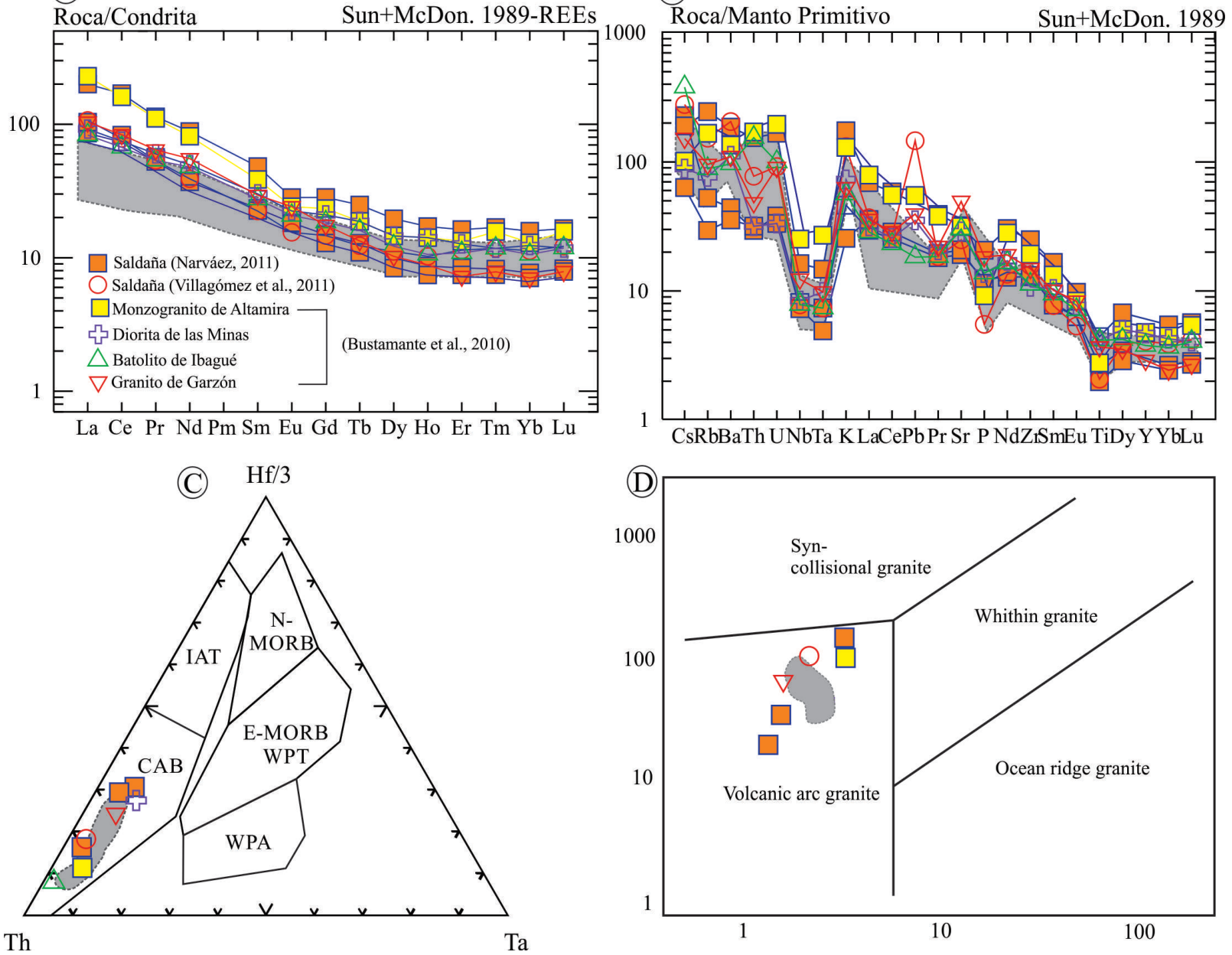

FIGURA 9. Gráficas comparativas de las diferentes unidades del Jurásico en Colombia con el Stock de Payandé (sombra gris). En todas las gráficas se observa claramente la similitud de los resultados de este trabajo con otros cuerpos ígneos. A.-B. Condrita normalizada con la composición de las tierras raras y distribución de los elementos traza normalizado con los valores de manto primitivo (Sun and McDonough, 1989). C. Diagrama de discrimación tectónica Th - Hf - Ta (Wood et al., 1980). D. Diagrama de discriminación tectónica de Pearce et al. (1984).

La similitud de los resultados geoquímicos del Stock de Payandé con los demás cuerpos plutónicos y volcanosedimentarios del Jurásicos es un elemento más para reevaluar la zona de rift propuesta para esta área (Jaillard et al., 1990 y Cediel et al., 2003) y aportar más evidencias a un modelo de arco magmático relacionado a una zona de subducción y cuencas sedimentarias trasarco el cual estaba funcionando durante el Jurásico Tardío en el VSM (Bayona et al., 1994; McCourt et al., 1984; Núñez, 1986; Toussaint, 1995; Vásquez et al., 2006; Sarmiento-Rojas et al., 2006; Bustamante et al., 2010; Spikings et al., 2014) 


\section{AGRADECIMIENTOS}

Expresamos nuestros sinceros agradecimientos a Mauricio Alvarán Echeverri, director del Semillero de Investigación en Metalografía y Petrografía por toda la asesoría en campo y revisión del manuscrito. Gracias por la colaboración en campo de Edwin Naranjo y Eliselder Zapata, a los docentes del programa de Geología de la Universidad de Caldas Luz Mary Toro Toro y Elvira Cristina Ruíz Jiménez por las ayudas en la parte analítica. Al doctor José María Jaramillo Mejía y al geólogo Andrés Naranjo Sierra por su colaboración en el proyecto, y por último y no menos importante a la Dra Nora Rubinstein de la Universidad de Buenos Aires y al revisor anónimo, por sus valiosos aportes para la mejora del manuscrito.

\section{REFERENCIAS}

Altenberger, U., and Concha, A. E. 2005. Late lower to early Middle Jurassic arc magmatism in the northern Ibagué-Batholith/Colombia. Geología Colombiana, 30: 87-97.

Alvarán, M., Naranjo, E., y Zapata, E. 2011. Skarn de cobre en la mina río frío, Payandé- Tolima: aspectos mineralógicos, metalográficos y microtermométricos. Boletín de Ciencias de la Tierra, 29: 7-20.

Bailey, J. C. 1981. Geochemical criteria for a refined tectonic discrimination of orogenic andesites. Chemical Geology, 32: 139-154.

Barrero, D. 1969. Petrografía del Stock de Payandé y metamorfitas asociadas. Boletín Geológico, 17: 113- 144.

Bayona, G., Rapalini, V., and Alvarez, V. 2006. Paleomagnetism in Mesozoic rocks of the northern Andes and its implications in Mesozoic tectonics of northwestern South America. Earth Planets Space, 58: 1-18.

Bayona, G., García, D., y Mora, G. 1994. La Formación Saldaña: producto de la actividad de estratovolcanes continentales en un dominio de retroarco, en EtayoSerna, F., ed., Estudios Geológicos del Valle Superior del Magdalena: Universidad Nacional de Colombia, Bogota, 121p.

Bayona, G., Silva, C., Rapalini, A. E., Costanzo-Álvarez, V., Aldana, M., y Roncancio, J. 2005. Paleomagnetismo y mineralogía magnética en rocas de la formación Saldaña y unidades cretácicas suprayacentes en la parte norte del Valle Superior del Magdalena, Colombia. Boletín de Geología, 27: 69-85.
Booden, M.A., Smith, I.E.M., Black, P.M., and Mauk, J.L. 2011. Geochemistry of the Early Miocene volcanic succession of Northland, New Zealand, and implications for the evolution of subduction in the Southwest Pacific. Journal of Volcanology and Geothermal Research, 199: 25-37.

Bustamante, C., Cardona, A., Bayona, G., Mora, A., Valencia, V., Gehrells, G., and Vervoort, J. 2010. U-Pb La-Icp-Ms geochronology and regional correlation of Middle Jurassic intrusive rocks from the Garzon Massif, Upper Magdalena Valley and Central Cordillera, Southern Colombia. Boletín de Geología, 32: 93-109.

Cediel, F., Shaw, R.P., and Cáceres, C. 2003. Tectonic assembly of the Northern Andean Block. In: Bartolini, C., Buffer, R.T., Blickwede, J. (Eds.), The Circum-gulf of Mexico and the Caribbean: Hydrocarbon Habitats, Basin Formation, and Plate Tectonics. AAPG memoir, 79, pp. 815-848.

Cediel, F., Mojica, J., y Macia, C. 1980. Definici6n estratiqráfica del triásico en Colombia, Suramerica, Formaciones Luisa, Payandé y Saldaña.- Newsletters Stratigraphic, 9: 73-104.

Davidson, J. P., Ferguson, K. M., Colucci, M. T., and Dungan, M. A. 1988. The origin and evolution of magmas from the San Pedro-Pellado volcanic complex, S. Chile: multicomponent sources and open systems evolution. Contribution to Mineralogy and Petrology, 100: 429-445.

Geyer, O. 1973. Das Prakretazische Mesozoikum Von Kolumbien. Geologishes Jahrbuch, 5:

1-156.

Gómez, J., Nivia, A., Montes, N. E., Jiménez, D .M., Sepúlveda, J., Gaona, T., Osorio, J. A., Diederix, H., Mora, M., y Velásquez, M. E., compiladores. 2007. Atlas Geológico de Colombia. Escala 1:500.000. INGEOMINAS, 26 planchas. Bogotá.

Harris, N. B., Pearce, J. A., and Tindle, A. G. 1986. Geochemical characteristics of collision- zone magmatism. Geological Society, London, Special Publications, 19: 67-81.

Hawkesworth, C. J., Gallagher, K., Hergt, J. M., and McDermott, F. 1994. Destructive plate margin magmatism: Geochemistry and melt generation. Lithos, $33: 169-188$ 
Hezarkhani, A. 2006. Petrology of the intrusive rocks within the Sungun porphyry copper deposit, Azerbaijan, Iran. Journal of Asian Earth Sciences, 27: 326-340.

Hirschmann, M.M., and Stolper, E.M. 1996. A possible role for garnet pyroxenite in the origin of "garnet signature" in MORB. Contribution to Mineralogy and Petrology, 124: 185-208.

Hubach, E. 1957. Contribución a las unidades estratigráficas de Colombia. INGEOMINAS, Bogotá Informe 1212: pp. 66.

Irvine, T.N., and Baragar, W.R.A. 1971. A guide to the chemical classification of the common volcanic rocks. Canadian Journal of Earth Sciences, 8: 523-548.

Jaillard, E., Soler, P., Carlier, G., and Mourier, T. 1990. Geodynamic evolution of the northern and central Andes during early to middle Mesozoic times: a Tethyan model. Journal of the Geological Society, 147:1009-1022.

Le Maitre, R. W. 2002. Igneous Rocks. A Classification and Glossary of Terms. Recommendations of the International Union of Geological Sciences Subcommission on the Systematics of Igneous Rocks, 2nd Ed. The Cambridge University. New York, 236p.

Long, L., Gao, J., Klemd, R., Beier, C., Qian, Q., Zhang, X., and Jiang, T. 2011. Geochemical and geochronological studies of granitoid rocks from the Western Tianshan Orogen: implications for continental growth in the southwestern Central Asian Orogenic Belt. Lithos, 126: 321-340.

Maniar, P.D., and Piccoli, P.M. 1989. Tectonic discrimination of granitoids. Geological Society of America Bulletin, 101: 635-643.

McCourt, W.J., Feininger, T., and Brook, M. 1984. New geological and geochronological data from the Colombian Andes: continental growth by multiple accretions. Journal of the Geological Society of London, 141: 831-845.

McCulloch, M.T., and Gamble, J.A. 1991. Geochemical and geodynamical constraints on subduction zone magmatism. Earth and Planetary Science Letters, 102: 358-374.

Middlemost, E. A. 1994. Naming materials in the magma/igneous rock system. Earth- Science Reviews, 37: 215-224.
Mojica, L. y Macía, C. 1982. Geología del extremo NE de la cuenca de Neiva (Valle Superior del Magdalena, región de Prado- Dolores, Tolima) XXI Annual Field Trip ACGGP, Vol. 1., Bogotá Colombia, pp. 45.

Mojica, J., Kammer, A., and Ujueta, G. 1996. El Jurásico del Sector Noroccidental de Suramérica y Guía de la Excursión al Valle Superior del Magdalena (Nov.14/95), Regiones de Payandé y Prado, Departamento del Tolima. Geología Colombiana, 21: 3-40.

Narváez, P. A. 2011. Caracterización Petrográfica y Geoquímica de la Formación Saldaña en los Sectores de los Municipios de Prado-Dolores (Departamento del Tolima) e inmediaciones del Río Venado (Departamento del Huila). Tesis de pregrado: Universidad de Caldas, Facultad de Ciencias Exactas y Naturales, departamento de Geología, 97p.

Nelson, H, W. 1959. Contribution to the geology of the Central and Western Cordillera of Colombia in the sector between Ibague and Cali. Leidsche Geol. Meded, 22: $1-75$.

Núñez, A. 2001. Memoria Explicativa del Departamento del Tolima. INGEOMINAS, 101p.

Núñez, A. 1986. Petrogénesis del batolito de Ibague. Geología Colombiana, 15: 35-45.

Ordóñez -Calderón, J. C., Polat, A., Fryer, B. J., and Gagnon, J. E. 2011. Field and geochemical characteristics of Mesoarchean to Neoarchean volcanic rocks in the Storø greenstone belt, SW Greenland: evidence for accretion of intra-oceanic volcanic arcs. Precambrian Research, 184: 24-42.

Pearce, J.A. 2008. Geochemical fingerprinting of oceanic basalts with applications to ophiolite classification and the search for Archean oceanic crust. Lithos, 100: 14-48.

Pearce J.A. 1983. The role of sub-continental lithosphere in magma genesis at destructive plate margins. In: Continental basalts and mantle xenoliths. Hawkesworth, C.J. and Norry, M.J. Shiva Publications Nantwich, United Kingdom, pp. 230-249.

Pearce, J. A., Harris, N. W., and Tindle, A. G. 1984. Trace element discrimination diagrams for the tectonic interpretation of granitic rocks. Journal of Petrology, 25: 956-983. 
Pindell, J., and Kennan, L. 2010. Tectonic evolution of the Gulf of Mexico, Caribbean and northern South America in the mantle reference frame: an update. In James, K. H., Lorente, M. A., and Pindell, J. eds., The Origin and Evolution of the Caribbean Plate: Geological Society of London Special Publication, 328: 1-56.

Pindell, J., and Dewey, J. 1982. Permo-Triassic reconstruction of western Pangaea and the evolution of the Gulf of Mexico-Caribbean region. Tectonics, 1: 179-211.

Pitcher, W.S., Atherton, M.P., Cobbing, E. J. and Beckinsale, A.D. 1985. Magmatism at a plate edge - The Peruvian Andes. Blackie, J. Wiley and Sons. London, 328p.

Ramos, V., and Alemán, A. 2000. Tectonic evolution of the Andes. In: Cordani, U. G., Milani, E. J., ThomazFilho, A, Campos, D. A. Tectonic evolution of South America. XXXI International Geological Congress, Rio de Janeiro, Brasil, pp. 635-685.

Sarmiento-Rojas, L. F., Van Wess, J. D., and Cloetingh, S. 2006. Mesozoic transtensional basin history of the Eastern Cordillera, Colombian Andes: Inferences from tectonic models. Journal of South American Earth Sciences, 21: 383- 411.

Siebe, C., Rodríguez- Lara, V., Schaaf, P., and Abrams, M. 2004. Geochemistry, Sr-Nd isotope composition, and tectonic setting of Holocene Pelado, Guasapala and Chichinautzin scoria cones, south of Mexico City. Journal of Volcanology and Geothermal Research, 130: 197-226.

Spikings, R., Cochrane, R., Villagómez, D., Van der Lelij, R., Vallejo, C., Winkler, W., and Beate, B. 2014. The geological history of northwestern South America: from Pangaea to the early collision of the Caribbean Large Igneous Province (290-75Ma). Gondwana Research, 27: 95-139.

Sun, S.S., and McDonough, W.F., 1989. Chemical and isotopic systematics of oceanic basalts: implications for mantle composition and processes. In: Saunders A D, Norry M (eds) Magmatism in Ocean Basins. Geological Society of London Special Publication, 42: 313-345.

Toussaint, J.F. 1995. Evolución Geológica de Colombia. Triásico - Jurásico. Universidad Nacional de Colombia. Bogota, pp. 277.
Toussaint, J.-F., and Restrepo, J.J. 1994. The Colombian Andes during Cretaceous times. In: Salfity, J.A. (Ed.), Cretaceous Tectonics in the Andes. Earth Evol. Sci. Vieweg, Wiesbaden, pp. 61-100.

Trumpy, D. 1943. Pre-Cretaceous of Colombia. Geological Society of America Bulletin, 54: 1281-1304.

Van Westrenen, W., Blundy, J., and Wood, B. 2001. High field strength element/rare earth fractionation during partial melting in the presence of garnet: implications for identifications of mantle heterogeneities. Geochemistry, Geophysics, Geosystems, 2: Paper number 2000GC000133.

Vásquez, M., Bayona, G., and Romer, R.L. 2006. Geochemistry of Jurassic volcanic rocks of the northern Andes: Insights for the Mesozoic evolution of Northwestern Gondwana. In: Especiales, A.P., Meetings, G.S. (Eds.), Backbone of Americas - Patagonia to Alaska, Mendoza, Argentina, pp. 62.

Villagómez, D., Spikings, R., Magna, T., and Kammer, A. 2011. Geochronology, geochemistry and tectonic evolution of the Western and Central cordilleras of Colombia. Lithos, 125: 875- 896.

Walker, J. A., Patino, L. C., Carr, M. J., and Feigenson, M. D. 2001. Slab control over HFSE depletions in central Nicaragua. Earth and Planetary Science Letters, 192: 533-543.

Wilson, M. 2007. Igneous Petrogenesis. A global tectonic approack. Netherland, Springer, 466p.

Yang, H., Wen-chun, G., Zhao, G.C., Dong, Y., Xu, W.L., Ji, Z., and Yu, J. 2015. Late Triassic intrusive complex in the Jidong region, Jiamusi-Khanka Block, NE China: Geochemistry, zircon U-Pb ges, Lu-Hf isotopes, and implications for magma mingling and mixing. Lithos, 224: 143-159.

Trabajo recibido: febrero 11 de 2015

Trabajo aceptado: febrero 19 de 2016

Manuscrito publicado en internet: febrero 25 de 2016 